\title{
Breast tumor kinase (Brk/PTK6) plays a role in the differentiation of primary keratinocytes
}

\author{
J. Tupper • M. R. Crompton • A. J. Harvey
}

Received: 7 October 2010/Revised: 16 December 2010/Accepted: 29 December 2010/Published online: 15 January 2011

(C) The Author(s) 2011. This article is published with open access at Springerlink.com

\begin{abstract}
Breast Tumor Kinase (Brk/PTK6) has a relatively limited expression profile in normal tissue. Its expression is restricted to epithelial cells that are differentiating such as those in the epidermis, and Brk expression appears to be absent from proliferating cells in normal tissue. Also, there is now some evidence to suggest that Brk plays a functional role in the differentiation of the keratinocytes in the epidermis. We have, therefore, investigated the role that Brk/PTK6 plays in normal human primary keratinocytes by suppressing protein levels using RNA interference. We show that as primary human keratinocytes are induced to differentiate in vitro, Brk levels decrease. Decreasing Brk protein levels lead to an increase in the number of cells with a permeable plasma membrane, a decrease in epidermal growth factor receptor (EGFR) and a parallel increase in keratin 10 levels, but classical markers of apoptosis or terminal differentiation are not affected. We propose Brk, Keratin 10 and EGFR are co-regulated during differentiation and that manipulating Brk expression can influence the differentiation of normal primary human keratinocytes.
\end{abstract}

\footnotetext{
J. Tupper

School of Biological Sciences, Royal Holloway,

University of London, Egham, Surrey TW20 0EX, UK

M. R. Crompton

Academic Development Services, Royal Holloway, University of London, Egham, Surrey TW20 0EX, UK

\section{A. J. Harvey ( $\square)$}

Biosciences, Brunel Institute for Cancer Genetics and Pharmacogenomics, Brunel University,

Kingston Lane, Uxbridge, Middlesex TW20 0EX, UK

e-mail: amanda.harvey@brunel.ac.uk
}

Keywords Keratinocyte - Cell differentiation . Breast tumor kinase (Brk/PTK6)

\section{Introduction}

Keratinocyte differentiation is tightly controlled and involves a range of signals and events, including tyrosine kinase activation, that are important in regulation. Signaling downstream of the epidermal growth factor receptor (EGFR) in the presence of constitutively active AKT initiates the growth arrest and differentiation process in response to elevated calcium [1], while decreased EGFR levels and signaling are associated with terminal differentiation [14].

The breast tumor kinase (Brk/PTK6) is a known potentiator of EGF signaling $[8,9]$ and an inhibitor of Akt $[5,25]$ and, its suppression decreases proliferation of breast cancer cells [6]. Although originally identified in breast carcinomas [13], Brk's expression profile in normal tissue is relatively restricted. Brk and its mouse orthologue, Sik, are found in differentiating epithelia. Brk expression has been identified in epithelial cells of the GI tract [12], epidermis [19, 21], prostate [3], and oral mucosa [15].

In addition to the expression profile in differentiating epithelia, there is an evidence to suggest that Brk plays a functional role in keratinocyte differentiation. Brk overexpression in the keratinocyte cell line $\mathrm{HaCaT}$ results in moderately elevated expression of a known keratinocyte differentiation marker, keratin 10 [21]. Increased Brkassociated kinase activity was observed in $\mathrm{HaCaT}$ cells, induced to differentiate with calcium [21]. Similar results were seen in primary murine keratinocytes where activity of Sik increased rapidly following the calcium treatment [20]. 
We investigated the role that Brk plays in normal human primary keratinocytes by suppressing protein levels using RNA interference. We hypothesized that since differentiating agents can increase Brk activity, the suppression of Brk expression would alter primary keratinocyte differentiation in vitro.

\section{Materials and methods}

Cell culture

Normal human adult keratinocytes were purchased from TCS Cell Works, maintained in Epilife media supplemented with full supplement and antibiotics (Cascade Biologics Inc., USA) and used below passage ten.

For calcium differentiation studies, cells were plated at $37,500 \mathrm{~cm}^{-2} .24 \mathrm{~h}$ later medium was changed for medium plus $1.2 \mathrm{mM} \mathrm{Ca}^{2+}$ for 5 days, at which point cells displayed an altered morphology and decreased growth compared with controls.

Methylcellulose (MC) studies were performed according to Watt [23]. Briefly, $2 \times 10^{5}$ cells were suspended for $48 \mathrm{~h}$ in $2 \mathrm{ml}$ methylcellulose per well of a polyHEMAcoated 6-well plate (Sigma).

\section{RNA interference}

Cells were transfected with Brk-targeting (5'-GGUGAUU UCUCGAGACAACTT- $\left.3^{\prime}\right)$ or Control siRNA (5'-GGA CACCAUCAAGUGUUCGTT- $3^{\prime}$ ) (Dharmacon). Two transfections were performed $24 \mathrm{~h}$ apart on $10^{5}$ cells with the siRNA duplex $(120 \mathrm{nM})$ and Oligofectamine (Invitrogen) in OptiMEM medium using the manufacturer's guidelines.

\section{Trypan blue staining}

Cell viability was determined using trypan blue exclusion. Cells were mixed with the stain to a final trypan blue concentration of $0.1 \%$ and counted using an improved neubauer hemocytometer. Cells that appeared blue when visualized by light microscopy were classed as nonviable.

\section{Cell death ELISA}

Cell death levels were measured using a cell death detection ELISA (Roche).

\section{Western blotting}

Cells were lysed in $2 \times$ SDS-PAGE loading buffer and separated by SDS-PAGE. Equal loading was determined by coomassie-blue staining and the presence of housekeeping proteins. Separated proteins were transferred to nitrocellulose membranes by electro-blotting in Towbin buffer. Membranes were incubated with antibodies to GAPDH, $\beta$-actin (Abcam), EGFR, K10, involucrin (Sigma) and Brk (ICR-100) [9] and proteins visualized with an appropriate HRP-conjugated secondary antibody and chemiluminescence.

\section{Results and discussion}

Keratinocytes are one of the few sites of physiological Brk expression [20,21], however, Brk's role in these cells has yet to be fully characterized. In this study normal human keratinocytes were used as model for studying the role of Brk in human primary cells.

Primary non-immortalized keratinocytes were induced to differentiate by either increasing the calcium concentration of the medium or by resuspending the cells in methylcellulose. When cells were suspended in methylcellulose for $48 \mathrm{~h}$ or cultured in calcium for up to 5 days the levels of both Keratin 10 and involucrin increased. Brk protein levels decreased under these conditions suggesting that, as cells become more terminally differentiated Brk protein decreases (Fig. 1a).

To determine whether experimentally decreasing Brk expression resulted in differentiation, RNA interference was used to decrease Brk protein expression. Brk suppression (Fig. 1b) resulted in an increase in the percentage of cells incorporating trypan blue both 24 and $48 \mathrm{~h}$ following the second transfection compared with control transfectants (Fig. 1c). This increase was seen with two different Brk-targeting siRNA sequences (data not shown).

Membrane permeabilization to trypan blue could be regarded as a sign of cell death, however, it is also an early sign of keratinocyte differentiation. Western blot analysis revealed that on Brk suppression, Keratin 10 levels increased (Fig. 1b), although there were no observable changes in levels of the differentiation markers Keratin 1 and involucrin, or activation markers Keratin 6 and 16 (data not shown).

The effects of Brk-suppression on cell membrane integrity coupled with an increase in Keratin 10 suggest that Brk suppression may contribute to primary keratinocyte differentiation. However, as levels of other differentiation markers were unaffected, it is likely that downregulation of Brk may be an early/intermediate event in differentiation and is not a sufficient signal alone to fully mediate the differentiation process. Activation status could be important, although Brk expression is differential in epidermal layers [20, 21].

The level of cytosolic, histone-bound DNA was determined by DNA ELISA as a measure of chromatin 
(a)

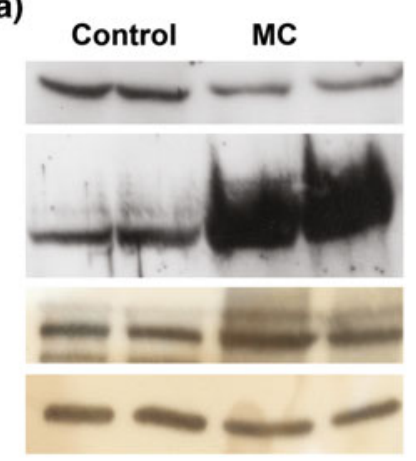

(b)

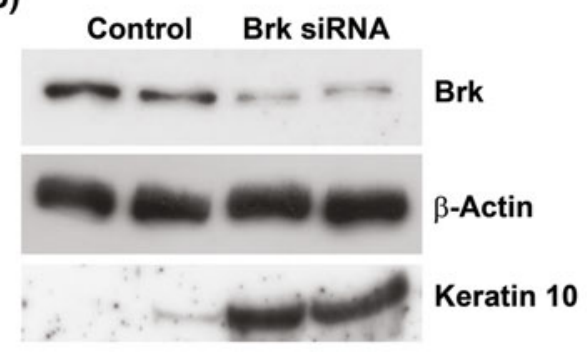



(c)

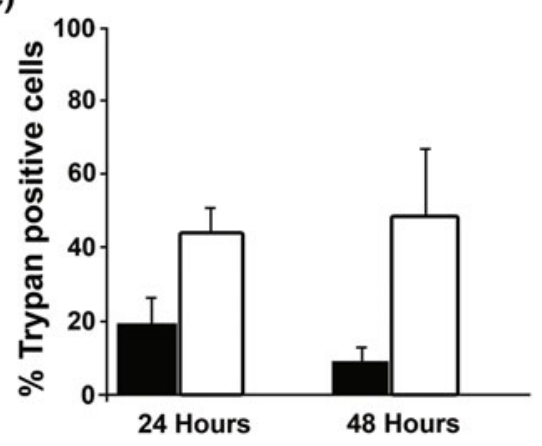

Fig. 1 Brk levels are reduced in differentiation. a Normal human primary keratinocytes were induced to differentiate by either culturing in methylcellulose for $48 \mathrm{~h}$ (MC) (left hand panel) or in medium containing $1.2 \mathrm{mM}$ Calcium ions for 5 days (Calcium) (right hand panel). Control cells were cultured under normal adherent culture conditions (Control). Cells were lysed in hot SDS-PAGE lysis buffer and relative protein levels determined by western blotting. Brk levels were then suppressed in normal human primary keratinocytes by
RNAi. b $48 \mathrm{~h}$ following the second transfection with control (Control) or Brk-targeting siRNA (Brk siRNA), cells were lysed in hot SDS-PAGE lysis buffer and relative protein levels determined by western blotting. c 24 and $48 \mathrm{~h}$ following the second transfection with control (filled bars) or Brk-targeting siRNA (open bars) cells were harvested, resuspended in $0.1 \%$ trypan blue and the percentage of nonviable cells determined

methylcellulose is much greater than that observed on Brk suppression. It is probable that methylcellulose culture affects a number of cellular pathways and processes involved in differentiation and that Brk contributes to this. However, it is unlikely that Brk is the only contributor to differentiation, which would account for the lesser effects in Brk-suppressed cells.

We have previously reported that Brk expression protected breast cancer cells from suspension-induced cell death [7]. Given that in vivo, Brk expression is increased in differentiating cells that are in transit away from the epidermal basement membrane, and that untransformed cells are dependent on contact with basement membrane/substratum for survival [4], we hypothesize that a function of Brk in early differentiating cells may be to promote their survival. The subsequent decrease in Brk levels that occurred when keratinocytes continued to differentiate might occur as a requirement of the differentiation process in primary cells. The expression of cell surface receptors associated with Brk signaling, such as EGFR, has been shown to decrease in vivo as cells commit to terminal differentiation [11, 14]. Inhibition of EGFR signaling matin fragmentation observed when cells were cultured on 
(a)

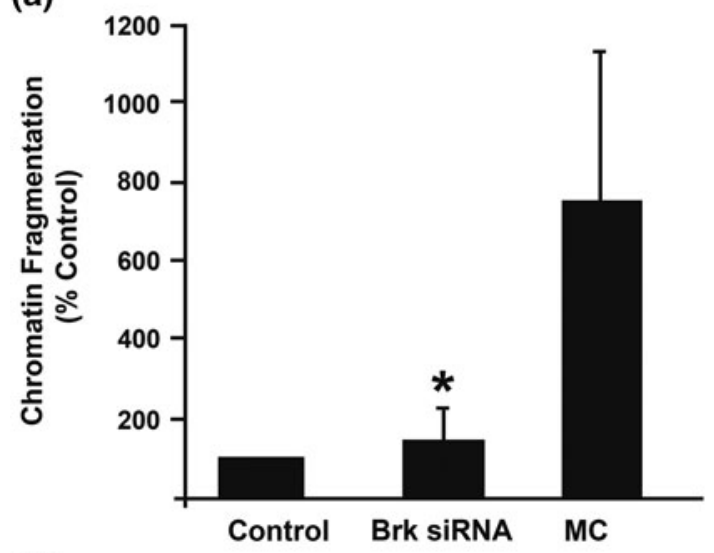

(b)

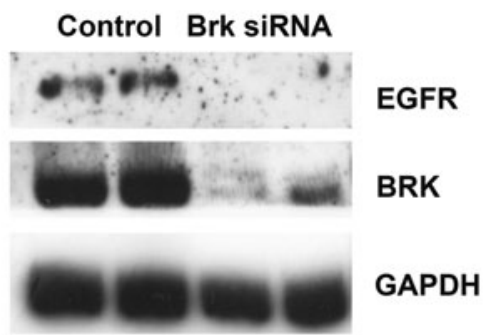

Fig. 2 Brk suppression induces chromatin fragmentation and suppression of EGFR. Brk levels were suppressed in normal human primary keratinocytes by RNAi. a $48 \mathrm{~h}$ following the second transfection with control (Control) or Brk-targeting siRNA (Brk siRNA), cells were harvested and assayed for chromatin fragmentation. As a positive control, primary keratinocytes were also cultured in methyl cellulose (MC) for $48 \mathrm{~h}$ prior to harvesting and assaying with the cell death ELISA. ${ }^{*} P=0.036$. b Parallel wells were also lysed in hot SDS-PAGE lysis buffer and relative protein levels determined by western blotting

induces growth arrest and keratinocyte differentiation [16]. As Brk enhances the effects of signaling via Erb receptors $[8,9]$ suppression of Brk would be predicted to reduce the effects of Erb signaling, in which this cellular context results in a more differentiated phenotype. Conversely EGF treatment of a serum-free epidermal culture model resulted in decreased differentiation [2] and an elevated expression of EGFR in differentiating cells, and presumably therefore activation of EGFR signaling, resulted in hyperproliferation [22]. Downregulation of EGFR signaling appears to be a requirement of keratinocyte differentiation and the reduction in signaling could be enhanced by reduced Brk levels. We propose that this may be a supplementary mechanism that contributes to the subsequent differentiation. In addition, we found that EGFR protein levels were decreased on Brk suppression in primary keratinocytes (Fig. 2b), which supports recent findings that Brk inhibits EGFR downregulation [10]. Brk suppression in keratinocytes may, therefore, be a mechanistic prerequisite for reduced EGFR levels in differentiation.

\section{Conclusions}

This is the first study in normal primary human cells demonstrating co-regulation of Brk and keratin 10 in differentiation. The data presented link Brk mechanistically to keratinocyte differentiation (via Keratin 10 and EGFR expression) and demonstrate that manipulating Brk affected phenotypic outcome. The results of this and other studies [20, 21] suggest that Brk plays an important, complex role in normal human keratinocyte differentiation and that this role could be keratinocyte-specific.

Acknowledgments This work was supported by a project grant from the Biotechnology and Biological Sciences Research Council, UK. The funder has played no role in the study design; in the collection, analysis, and interpretation of data; in the writing of the report; and in the decision to submit the paper for publication.

Open Access This article is distributed under the terms of the Creative Commons Attribution Noncommercial License which permits any noncommercial use, distribution, and reproduction in any medium, provided the original author(s) and source are credited.

\section{References}

1. Calautti E, Li J, Saoncella S, Brissette JL, Goetinck PF (2000) Phosphoinositide 3-kinase signaling to Akt promotes keratinocyte differentiation versus death. J Biol Chem 280(38):32856-32865

2. Chen CS, Lavker RM, Rodeck U, Risse B, Jensen PJ (1995) Use of a serum-free epidermal culture model to show deleterious effects of epidermal growth factor on morphogenesis and differentiation. J Invest Dermatol 104(1):107-112

3. Derry JJ, Richard S, Valderrama Carvajal H, Ye X, Vasioukhin V, Cochrane AW, Chen T, Tyner AL (2000) Sik (BRK) phosphorylates Sam68 in the nucleus and negatively regulates its RNA binding ability. Mol Cell Biol 20(16):6114-6126

4. Frisch SM, Francis H (1994) Disruption of epithelial cell-matrix interactions induces apoptosis. Cell Biol 24(4):619-626

5. Haegebarth A, Perekatt AO, Bie W, Gierut JJ, Tyner AL (2009) Induction of protein tyrosine kinase 6 in mouse intestinal crypt epithelial cells promotes DNA damage-induced apoptosis. Gastroenterology 137(3):945-954

6. Harvey AJ, Crompton MR (2003) Use of RNA interference to validate Brk as a novel therapeutic target in breast cancer: Brk promotes breast carcinoma cell proliferation. Oncogene 22:5006-5010

7. Harvey AJ, Pennington C, Porter S et al (2009) Brk protects breast cancer cells from autophagic cell death induced by loss of anchorage. Am J Pathol 175(3):1226-1234

8. Kamalati T, Jolin HE, Fry MJ, Crompton MR (2000) Expression of the Brk tyrosine kinase in mammary epithelial cells enhances the coupling of EGF signaling to PI 3-kinase and Akt, via erbB3 phosphorylation. Oncogene 19(48):5471-5476

9. Kamalati T, Jolin HE, Mitchell PJ et al (1996) Brk, a breast tumor-derived non-receptor protein-tyrosine kinase, sensitizes mammary epithelial cells to epidermal growth factor. J Biol Chem 271(48):30956-30963

10. Kang SA, Lee ES, Yoon HY et al (2010) PTK6 inhibits downregulation of EGF receptor through phosphorylation of ARAP1. J Biol Chem 285(34):26013-26021 
11. King LE Jr, Gates RE, Stoscheck CM, Nanney LB (1990) The EGF/TGF alpha receptor in skin. J Invest Dermatol 94:164S$170 \mathrm{~S}$

12. Llor X, Serfas MS, Bie W, Vasioukhin Y, Polonskaia M, Derry J, Abbott CM, Tyner AL (1999) BRK/Sik expression in the gastrointestinal tract and in colon tumors. Clin Cancer Res 5(7): 1767-1777

13. Mitchell PJ, Barker KT, Martindale JE, Kamalati T, Lowe PN, Page MJ, Gusterson BA, Crompton MR (1994) Cloning and characterisation of cDNAs encoding a novel non-receptor tyrosine kinase, brk, expressed in human breast tumours. Oncogene 9(8):2383-2390

14. Nanney LB, Stoscheck CM, King LE Jr et al (1990) Immunolocalization of epidermal growth factor receptors in normal developing human skin. J Invest Dermatol 94(6):742-748

15. Petro BJ, Tan RC, Tyner AL, Lingen MW, Watanbabe K (2004) Differntial expression of the non-receptor tyrosine kinase BRK in oral squamous cell carcinoma and normal oral epithelium. Oral Oncol 40(10):1040-1047

16. Peus D, Hamacher L, Pittelkow MR (1997) EGF-receptor tyrosine kinase inhibition induces keratinocyte growth arrest and terminal differentiation. J Invest Dermatol 109:751-756

17. Polakowska RR, Piacentini M, Bartlett R et al (1994) Apoptosis in human skin development: morphogenesis, periderm, and stem cells. Dev Dyn 199:176-188
18. Sun TT, Green H (1976) Differentiation of the epidermal keratinocyte in cell culture: formation of the cornified envelope. Cell 9:511-521

19. Vasioukhin V, Serfas MS, Siyanova EY et al (1995) A novel intracellular epithelial cell tyrosine kinase is expressed in the skin and gastrointestinal tract. Oncogene 10(2):349-357

20. Vasioukhin V, Tyner AL (1997) A role for the epithelial-cellspecific tyrosine kinase Sik during keratinocyte differentiation. Proc Natl Acad Sci 94(26):14477-14482

21. Wang TC, Jee SH, Tsai TF et al (2005) Role of breast tumor kinase in the in vitro differentiation of $\mathrm{HaCaT}$ cell. $\mathrm{Br} \mathrm{J}$ Dermatol 153(2):282-289

22. Wang X, Bolotin D, Chu DH, Polak L, Williams T, Fuchs E (2006) AP-2 $\alpha$ : a regulator of EGF receptor signalling and proliferation in skin epidermis. J Cell Biol 172(3):409-421

23. Watt FM (1994) In: Leigh, Watt (eds) Keratinocyte Methods, Cambridge University Press

24. Weil M, Raff MC, Braga VM (1999) Caspase activation in the terminal differentiation of human epidermal keratinocytes. Curr Biol 9(7):361-364

25. Zhang P, Ostrander JH, Faivre EJ et al (2005) Regulated association of protein kinase B/Akt with breast tumour kinase. J Biol Chem 280(3):1982-1991 